\title{
Hohe Berufsidentifikation trotz steigender Bürokratie
}

Die Bürokratie wird weiter zunehmen - das war die Hauptsorge der Ärzteschaft vor der Einführung von SwissDRG. Diese Befürchtung hat sich nun bestätigt. Jedoch geht der administrative Mehraufwand nicht zulasten der Patientinnen und Patienten; im Durchschnitt verbringen die Ärzte praktisch gleich viel Zeit mit ihnen. Die steigenden administrativen Aufgaben werden vielmehr durch zusätzliche Überstunden bewältigt. Trotzdem identifizieren sich die Ärztinnen und Ärzte stark mit ihrem Beruf und sind mehrheitlich zufrieden mit ihrer Tätigkeit. Als problematisch beurteilen sie beispielsweise den Einfluss der Krankenkassen auf die Behandlung: Durch schleppende Kostengutsprachen werden Überweisungen in die Rehabilitation verzögert.

\begin{abstract}
Beatrix Meyera,
Lukas Golder ${ }^{b}$,

Claude Longchampc

a Leiterin Tarife und Gesundheitsökonomie Spitalärzte

b Senior-Projektleiter, gfs.bern

c Institutsleiter, gfs.bern
\end{abstract}

Korrespondenz: FMH

Froburgstrasse 15

CH-4600 Olten

Tel. 0313591111

Fax 0313591112

tarife.spital[at]fmh.ch
Seit zwei Jahren rechnen die akutsomatischen Spitäler im stationären Bereich ihre Leistungen mit dem Fallpauschalensystem SwissDRG ab. Doch welche Auswirkungen haben SwissDRG und weitere Änderungen der neuen Spitalfinanzierung? Und wie ist die aktuelle Situation in der stationären Psychiatrie und Rehabilitation? Für diese Bereiche werden bis 2018 ebenfalls leistungsbezogene Tarifsysteme eingeführt. Um Fehlentwicklungen frühzeitig zu erkennen, führt das Forschungsinstitut gfs.bern im Auftrag der FMH seit 2011 repräsentative Befragungen durch. An der jüngsten Umfrage im Sommer 2013 haben über 1500 Ärztinnen und Ärzte teilgenommen. Das Ergebnis der Befragungen 2011 bis 2013 wird hier zusammenfassend dargestellt [1].

\section{Zunehmende Bürokratie durch Überstunden bewältigt}

Die grosse Sorge der Ärzteschaft vor drei Jahren war die weitere Zunahme der Bürokratie. Diese Befürchtung hat sich nun bestätigt. Innerhalb von drei Jahren hat der Aufwand für das Patientendossier und die Dokumentationsarbeit in der Akutsomatik durchschnittlich um fast 20 Minuten pro Woche zugenommen (Abb. 1). Eine gute Dokumentation ist unter SwissDRG notwendig, damit die Leistungen korrekt kodiert und vergütet werden können. Der administrative Mehraufwand erfolgte jedoch nicht zulasten der Patienten. Die Ärzte wollen nicht weniger Zeit für ihre Patienten zur Verfügung haben und nehmen Überstunden in Kauf, um die administrativen Arbeiten zu bewältigen. Im Jahr 2013 sind es in den akutsomatischen Spitälern 8,1 Überstunden pro Woche und damit deutlich mehr als in den Vorjahren. Konstant ein Drittel der Akutsomatiker findet die allgemeine Arbeitsorganisation im Spital schlecht.
Auch in der stationären Psychiatrie und Rehabilitation ist der administrative Aufwand beträchtlich. Jedoch hat die Arbeit am Patientendossier zwischen 2011 und 2013 nicht markant zugenommen. Dafür wird in der Psychiatrie und Rehabilitation anteilsmässig mehr Zeit mit dem Beantworten von Nachfragen der Krankenkassen und mit dem Einholen von Kostengutsprachen verbracht als in der Akutsomatik.

\section{Konstant hohe Berufsidentifikation}

Die Identifikation mit dem Beruf und die Arbeitszufriedenheit bleiben in der Akutsomatik insgesamt praktisch gleich hoch wie in den letzten beiden Jahren. 94\% der akutsomatischen Spitalärzte finden ihre Arbeit interessant und abwechslungsreich. 82\% sind sehr oder eher zufrieden mit ihrer Tätigkeit. Klar unzufriedener als in den Vorjahren sind allerdings die Assistenzärzte: $23 \%$ äusserten sich im Jahr 2013 sehr oder eher unzufrieden mit ihrer Arbeit, im Jahr 2011 waren es 17\%. Über eine Stelle ausserhalb des schweizerischen Gesundheitswesens denken $11 \%$ aller akutsomatischen Spitalärzte nach. In der Psychiatrie sind $82 \%$ und in der Rehabilitation $86 \%$ mit ihrer Arbeitstätigkeit sehr oder eher zufrieden.

\section{Teamarbeit hat nicht gelitten}

Die Zusammenarbeit im Team und mit den praxisambulant tätigen Ärzten hat in den letzten Jahren nicht gelitten. Nach wie vor sind über $90 \%$ der Akutsomatiker zufrieden mit ihrem eigenen Team. Unverändert geben exakt $76 \%$ der akutsomatischen Spitalärzte an, dass die Zusammenarbeit mit den praxisambulanten Ärzten gut oder sehr gut funktioniert. Wie in den Vorjahren bezeichnet jedoch nur eine Minderheit der praxisambulant tätigen Ärzte 
Abbildung 1

Zeitaufwand der Ärzte in der Akutsomatik für verschiedene Tätigkeiten.

«Wenn Sie an den letzten normalen Arbeitstag denken, wie viel Zeit haben Sie dabei auf die folgenden Tätigkeiten angewendet? Bitte geben sie ihre Angaben in Minuten an.»

in Mittelwerten akutsomatische Ärzte, in Minuten / Arbeitstag

medizinische patientennahe Tätigkeiten

$$
\begin{gathered}
\text { ärztliche } \\
\text { Dokumentationsarbeit/Patientendossier }
\end{gathered}
$$

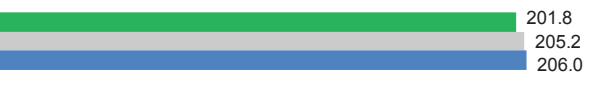

Visiten

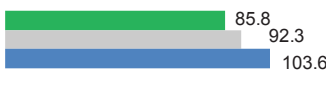

103.6

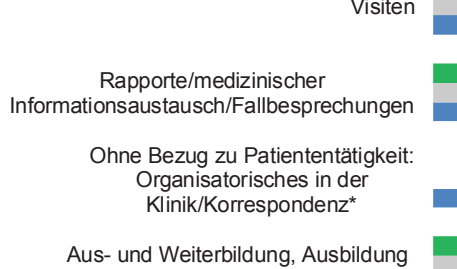

KollegInnen, Literaturstudium

sonstige administrative Tätigkeiten

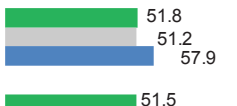

2011

2012

$-2013$
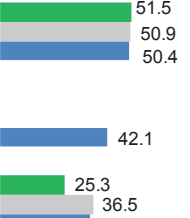

36.5

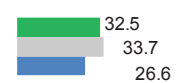

26.6

๑ gfs.bern, Begleituntersuchung SwissDRG im Auftrag der FMH, Juni - August 2013 (N akutsomatische Ärzte jeweils ca. 1000)

* 2013 das erste Mal befragt die Zusammenarbeit mit den Spitalärzten als sehr gut oder gut. Probleme werden zunehmend in der Zusammenarbeit mit den Rehabilitationskliniken festgestellt.

\section{Ärzte nehmen sich gleich viel Zeit für ihre Patienten - die zunehmende Bürokratie wird durch Überstunden bewältigt.}

ten in ihrem Arbeitsumfeld durchschnittlich 5,5 Mal pro Monat fest, dass Krankenkassen eine Überweisung in die Rehabilitationskliniken verzögerten. Aufgrund des Fallpauschalensystems in der stationären Akutsomatik und den Tagespauschalen in der stationären Rehabilitation besteht für die Krankenkassen ein Anreiz für diese Verzögerungen. Effiziente akutsomatische Kliniken werden dadurch bestraft. Problematisch sind diese Verzögerungen hauptsächlich für die Patienten, denn eine frühzeitige Rehabilitation ist zentral für das Behandlungsergebnis. Diese Praktiken der Krankenkassen gilt es zu unterbinden. Die FMH prüft, wie hier Gegensteuer gegeben werden kann.

\section{Gute Versorgung - verschiedene Fehlentwicklungen}

Eine deutliche Mehrheit der Spitalärzte bewertet den Versorgungsstandard in ihrem unmittelbaren Arbeitsbereich als sehr gut oder eher gut. Zunehmend beobachtet die akutsomatische Ärzteschaft jedoch, dass versucht wird, chronisch kranke und multimorbide Patienten in anderen Einrichtungen unterzubringen: Für 33\% der akutsomatischen Ärzte trifft diese Aussage auf ihre Abteilung oder Klinik sehr oder eher zu. Zwar eher selten, aber konstant zunehmend ist die Aufteilung der Behandlung auf mehrere Spitalbesuche bei multimorbiden Patienten. Etwas häufiger als noch vor einem Jahr kommt es vor, dass die Entscheidung, ob eine Behandlung spitalambulant oder stationär durchgeführt werden soll, aus nicht-medizinischen Gründen gefällt wird.

\section{Kritische Beobachtung notwendig}

Die FMH hat diese Ergebnisse bereits ausführlich diskutiert mit ihren internen Organen: FMH-Fachkommission SwissDRG, den FMH-Begleitgruppen ST Reha und TARPSY, im Zentralvorstand und in der Delegiertenversammlung. Zentral ist, dass die Entwicklung der Qualität, der Bürokratie sowie des Einflusses der Krankenkassen auch künftig kritisch beobachtet werden. Noch nehmen die Ärzte aufgrund ihrer hohen Berufsidentifikation überdurchschnittliche Belastungen in Kauf. Noch leisten die Ärzte Überstunden, um trotz steigender Bürokratie genügend Zeit für ihre Patienten zu haben und um diese qualitativ gut behandeln zu können. Doch die Ärzteschaft kann Fehlentwicklungen nicht unbegrenzt durch Mehrarbeit auffangen. Vielmehr sind von den Spitälern Massnahmen zur administrativen Entlastung der Ärzte einzuleiten, damit sich diese auf die Behandlung ihrer Patienten konzentrieren können.

1 Zur ausführlichen Studie vgl. Golder L et al. Gute Patientenversorgung trotz administrativem Aufwand punktuelle Probleme. Schlussbericht 2013. www.fmh.ch $\rightarrow$ Stationäre Tarife $\rightarrow$ Begleitforschung $\rightarrow$ Entwicklung Rahmenbedingungen Ärzteschaft. 